Скопје, Македонија

\title{
A CHARACTERIZATION OF STRICTLY CONVEX 2-NORMED SPACE
}

\author{
Samoil Malčeski ${ }^{1}$, Katerina Anevska ${ }^{2}$ and Risto Malčeski ${ }^{3}$
}

\begin{abstract}
The terms of 2-norm and 2-normed are given by S. Gähler in the paper [10], Ch. Diminnie, S. Gahler and A. White ([3]) gave the term of strictly convex 2-normed spaces. This paper consists of several characterizations of strictly convex 2-normed spaces.
\end{abstract}

\section{INTRODUCTION}

Let $L$ be a real vector space with dimension greater than 1 and $\|\cdot, \cdot\|$ be a real function on $L \times L$ such that:

a) $\|x, y\|=0$ if and only if the set $\{x, y\}$ is linearly dependent;

b) $\|x, y\|=\|y, x\|$, for all $x, y \in L$;

c) $\|\alpha x, y\|=|\alpha| \cdot\|x, y\|$, for all $x, y \in L$ and for each $\alpha \in \mathbf{R}$,

d) $\|x+y, z\| \leq\|x, z\|+\|y, z\|$, for all $x, y, z \in L$.

The function $\|\cdot \cdot \cdot\|$ is said a 2-norm on $L$, and $(L,\|\cdot, \cdot\|)$ is said a vector 2normed space ([10]). Some of the fundamental properties of the 2-norm are the following:

1. $\|x, y\| \geq 0$, for all $x, y \in L$ and

2. $\|x, y+\alpha x\|=\|x, y\|$, for all $x, y \in L$ and for each $\alpha \in \mathbf{R}$.

Let $n>1$ be a positive integer, $L$ be a real vector space, $\operatorname{dim} L \geq n$ and $(\cdot, \cdot \mid \cdot)$ be a real function on $L \times L \times L$ such that

i) $(x, x \mid y) \geq 0$, for all $x, y \in L$ and $(x, x \mid y)=0$ if and only if $x$ and $y$ are linearly dependent;

ii) $(x, y \mid z)=(y, x \mid z)$, for all $x, y, z \in L$,

iii) $(x, x \mid y)=(y, y \mid x)$, for all $x, y \in L$;

2010 Mathematics Subject Classification. Primary: 46B20 Secondary: 46C05

Key words and phrases. strictly convex space, end point, minimal point with respect to a set 
iv) $(\alpha x, y \mid z)=\alpha(x, y \mid z)$, for all $x, y, z \in L$ and for each $\alpha \in \mathbf{R}$; and

v) $\left(x+x_{1}, y \mid z\right)=(x, y \mid z)+\left(x_{1}, y \mid z\right)$, for all $x, x_{1}, y, z \in L$.

The function $(\cdot, \cdot \mid \cdot)$ is said a 2-inner product, and $(L,(\cdot, \cdot \mid \cdot))$ is said a 2-preHilbert space ([1]).

The concepts of 2-norm and 2-inner product are two-dimensional analogous to the concepts of a norm and an inner product. R. Ehret proved ([7]), that if $(L,(\cdot, \cdot \mid \cdot))$ is a 2-pre-Hilbert space, then $\|x, y\|=(x, x \mid y)^{1 / 2}$ defines a 2-norm. Thus, we get a vector 2 -normed space $(L,\|\cdot, \cdot\|)$ and moreover, for all $x, y, z \in L$ it is true that

$$
\begin{gathered}
(a, b \mid c)=\frac{\|a+b, c\|^{2}-\|a-b, c\|^{2}}{4}, \\
\|x+y, z\|^{2}+\|x-y, z\|^{2}=2\left(\|x, z\|^{2}+\|y, z\|^{2}\right),
\end{gathered}
$$

The equality (2) is actually two-dimensional analogy to the parallelogram equality and it is said parallelepiped equality. Further, if $(L,\|\cdot, \cdot\|)$ is a vector 2normed space such that for all $x, y, z \in L$, (1) holds true, then (2) defines 2inner product on $L$, whereby for all $a, b \in L,\|x, y\|=(x, x \mid y)^{1 / 2}$, holds true

Let $z$ be a fixed non-null element of $L, V(z)$ be a subspace of $L$ generated by $z$ and $L_{z}$ be a factor space $L / V(z)$. Let $x_{z}$ be the class of equivalence of $x$ with respect to $V(z)$. Clearly, $L_{z}$ is a vector space in which the operations vector addition and scalar multiplication are defined as following $x_{z}+y_{z}=(x+y)_{z}$ and $\alpha x_{z}=(\alpha x)_{z}$. In [6] is proven that $\left\|x_{z}\right\|_{z}=\|x, z\|$ defines a norm on $L_{z}$.

Let $x, y \in L$ be non-null elements and let $V(x, y)$ be the subspace of $L$ generated by the vectors $x$ and $y$. The vector 2 -normed space $(L,\|\cdot, \cdot\|)$ is called a strictly convex if $\|x, z\|=\|y, z\|=\left\|\frac{x+y}{2}, z\right\|=1$ and $z \notin V(x, y)$, for $x, y, z \in L$, implies $x=y$ ([3]). Several characterizations of strictly convex 2normed space are given in papers [1], [5] - [8], [12] - [14], [16], [17], [23] and [24], and a few of them will be stated in the following theorem.

Theorem 1. Let $(L,\|\cdot, \cdot\|)$ be a 2-normed space. The following claims are equivalent:

1) $(L,\|\cdot, \cdot\|)$ is strictly convex

2) For any non-null $z \in L$, the space $L_{z}$ is strictly convex 
3) If $\|x+y, z\|=\|x, z\|+\|y, z\|$ and $z \notin V(x, y)$, for $x, y, z \in L$ then $y=\alpha x$ for some $\alpha>0$.

4) If $\|x-u, z\|=\alpha\|x-y, z\|,\|y-u, z\|=(1-\alpha)\|x-y, z\|, \quad \alpha \in(0,1)$ and $z \notin V(x-u, y-u)$, then $u=(1-\alpha) x+\alpha y$.

$5)$ if $\|x, z\|=y, z \|=1, \quad x \neq y$ and $z \notin V(x, y)$, for $x, y, z \in L$, then $\left\|\frac{x+y}{2}, z\right\|<1$.

Example 1 ([3]). Let $L$ be 2-preHilber space and $x, y, z \in L$ be such that $\|x, z\|=$ $\|y, z\|=1, x \neq y$ and $z \notin V(x, y)$. Then the parallelepiped equality implies

$$
\left\|\frac{x+y}{2}, z\right\|^{2}+\left\|\frac{x-y}{2}, z\right\|^{2}=1 \text {. }
$$

But, $x \neq y$ and $z \notin V(x, y)$, thus $\left\|\frac{x-y}{2}, z\right\|>0$ and the equality (3) implies $\left\|\frac{x+y}{2}, z\right\|<1$. Finally, thereby Theorem $1, L$ is strictly convex.

Example 2 ([18]). In the set of bounded series of real numbers $l^{\infty}$

$$
\|x, y\|=\sup _{\substack{i, j \in \mathbf{N} \\
i<j}}|| \begin{array}{cc}
x_{i} & x_{j} \\
y_{i} & y_{j}
\end{array}||, x=\left(x_{i}\right)_{i=1}^{\infty}, y=\left(y_{i}\right)_{i=1}^{\infty} \in l^{\infty}
$$

defines a 2-norm. That is, $\left(l^{\infty},\|\cdot, \cdot\|\right)$ is a real 2-normed space. The vectors

$$
\begin{gathered}
x=\left(1-\frac{1}{2}, 1-\frac{1}{2^{2}}, \ldots, 1-\frac{1}{2^{n}}, \ldots\right), y=\left(0,1-\frac{1}{2}, 1-\frac{1}{2^{2}}, \ldots, 1-\frac{1}{2^{n-1}}, \ldots\right) \text { and } \\
z=(1,0,0, \ldots, 0, \ldots)
\end{gathered}
$$

satisfy $\|x, z\|=\|y, z\|=\left\|\frac{x+y}{2}, z\right\|=1$ and $z \notin V(x, y)$, but $x \neq y$. Therefore, $l^{\infty}$ is not strictly convex 2-normed space.

\section{MAINS ReSUltS}

Let $x, y \in L$. The set

$$
[x, y]=\{\alpha x+(1-\alpha) y \mid \alpha \in[0,1]\}
$$

is said a line segment (segment) with end points $x$ and $y$. The set

$$
(x, y)=\{\alpha x+(1-\alpha) y \mid \alpha \in(0,1)\}
$$

is said an opened line segment with end points $x$ and $y$. 
Corollary 1. Let $(L,\|\cdot, \cdot\|)$ be a 2-normed space. The following claims are equivalent:

1) $(L,\|\cdot, \cdot\|)$ is strictly convex

2) If $\|x+y, z\|=\|x, z\|+\|y, z\|$ and $z \notin V(x, y)$, for $x, y, z \in L$, then the set $[x, y]=\{\alpha x+(1-\alpha) y \mid \alpha \in[0,1]\}$ is linearly dependent.

Proof. Let the condition 2) holds true. Since $x, y \in[x, y]$, the set $\{x, y\}$ is linearly dependent, i.e. it exists $\alpha \in \mathbf{R}$ such that $y=\alpha x$. If we substitute in the condition $\|x+y, z\|=\|x, z\|+\|y, z\|$ and consider that $z \notin V(x, y)$, we get $|1+\alpha|=1+|\alpha|$, which implies $\alpha>0$. Thus, $y=\alpha x$ for some $\alpha>0$, so Theorem 1 implies that $L$ is strictly convex.

Let $L$ be a strictly convex space. If $\|x+y, z\|=\|x, z\|+\|y, z\|$ and $z \notin V(x, y)$, then Theorem 1 implies that $y=\alpha x$ for some $\alpha>0$. Let $x_{t}=t x+(1-t) y$, for $t \in[0,1]$. Thus, $x_{t}=(t+(1-t) \alpha) x$, for $t \in[0,1]$ and thereby all $t, p \in[0,1]$ satisfy $t+(1-t) \alpha>0$ and $p+(1-p) \alpha>0$ we get

$$
x_{t}=(t+(1-t) \alpha) x=\frac{t+(1-t) \alpha}{p+(1-p) \alpha}(p+(1-p) \alpha) x_{p}=\frac{t+(1-t) \alpha}{p+(1-p) \alpha} x_{p},
$$

So, the set $\left\{x_{t}, x_{p}\right\}$ is linearly dependent, for all $t, p \in[0,1]$. Thus, the set $[x, y]=\{\alpha x+(1-\alpha) y \mid \alpha \in[0,1]\}$ is linearly dependent.

Let $L$ be a 2-normed space, $x, z \in L$ and $r>0$. The set

$$
B_{z}(x, r)=\{y \in L \mid\|y-x, z\|<r\}
$$

is called an opened ball with respect to $z$ centered at $x$ and radius $r$. If $x=0$ and $r=1$, then $B_{z}(0,1)$ is called $a$ unit opened ball with respect to $z$. The set

$$
B_{z}[x, r]=\{y \in L \mid\|y-x, z\| \leq r\}
$$

is called $a$ closed ball with respect to $z$ centered at $x$ and radius $r$. If $x=0$ and $r=1$, then $B_{z}[0,1]$ is called a unit closed ball with respect to $z$. The set

$$
S_{z}(x, r)=\{y \in L \mid\|y-x, z\|=r\}
$$

is called $a$ sphere with respect to $z$ centered at $x$ and radius $r$. If $x=0$ and $r=1$, then $S_{z}(0,1)$ is called a unit closed sphere with respect to $z$. Clearly,

$$
B_{z}(x, r) \subseteq B_{z}[x, r] \text { and } B_{z}[x, r]=B_{z}(x, r) \cup S_{z}(x, r) .
$$

Lemma 1. Let $L$ be a 2-normed space and $x, y, z \in L$. If

$$
\|x+y, z\|=\|x, z\|+\|y, z\|,
$$


then, for all $t, s \geq 0$

$$
\|t x+s y, z\|=t\|x, z\|+s\|y, z\|,
$$

holds true. If $z \notin V(x, y)$, then $\left[\frac{x}{\|x, z\|}, \frac{y}{\|y, z\|}\right] \subseteq S_{z}(0,1)$.

Proof. Let $0 \leq s \leq t$. Then the properties of the 2-norm imply

$$
\begin{aligned}
t\|x, z\|+s \| y, z & \|\geq t x+s y, z\| \\
& =\|t(x+y)-(t-s) y, z\| \\
& \geq\|t(x+y), z\|-\|(t-s) y, z\| \\
& =t\|x+y, z\|-(t-s)\|y, z\| \\
& =t\|x, z\|+t\|y, z\|-t\|y, z\|+s\|y, z\| \\
& =t\|x, z\|+s\|y, z\|,
\end{aligned}
$$

i.e. in this case the equality (4) holds true. Analogously can be considered a case for $0 \leq t \leq s$.

Let $z \notin V(x, y)$. If $\alpha \in[0,1]$, then the equality (4) implies

$$
\left\|\alpha \frac{x}{\|x, z\|}+(1-\alpha) \frac{y}{\|y, z\|}, z\right\|=\frac{\alpha}{\|x, z\|}\|x, z\|+\frac{1-\alpha}{\|y, z\|}\|y, z\|=1 .
$$

Therefore, $\left[\frac{x}{\|x, z\|}, \frac{y}{\|y, z\|}\right] \subseteq S_{z}(0,1)$.

Theorem 2. The 2-normed space $L$ is strictly convex if and only if $\|x, z\|=\|y, z\|=1$ and $[x, y] \subseteq S_{z}(0,1)$ imply $x=y$.

Proof. Let $L$ be a strictly convex 2-normed space and let the conditions $\|x, z\|=\|y, z\|=1$ and $[x, y] \subseteq S_{z}(0,1)$ be satisfied. Then, $[x, y] \subseteq S_{z}(0,1)$, for $\alpha=\frac{1}{2}$ implies $\frac{x+y}{2}=\frac{1}{2} x+\left(1-\frac{1}{2}\right) y \in S_{z}(0,1)$. That is $\left\|\frac{x+y}{2}, z\right\|=1$. Moreover, since $L$ is strictly convex, we get that $x=y$.

Conversely, let $\|x, z\|=\|y, z\|=1$ and $[x, y] \subseteq S_{z}(0,1)$ imply $x=y$. Let's assume that $\|x+y, z\|=\|x, z\|+\|y, z\|$ and $z \notin V(x, y)$. Thus, Lemma 1 implies $\left[\frac{x}{\|x, z\|}, \frac{y}{\|y, z\|}\right] \subseteq S_{z}(0,1)$, which according to assumption means $\frac{x}{\|x, z\|}=\frac{y}{\|y, z\|}$, i.e. $x=\frac{\|x, z\|}{\|y, z\|} y$. Finally, the Theorem 1 implies $L$ is strictly convex 2-normed space.

Theorem 3. A 2-normed space $L$ is strictly convex if and only if the following condition is satisfied 


$$
\begin{aligned}
& x, y \in S_{z}(0,1), x \neq y \text { implies } \\
& \|\alpha x+\beta y, z\|<1, \text { for } \alpha, \beta>0 \text { and } \alpha+\beta=1 .
\end{aligned}
$$

Proof. Let the condition (5) be satisfied and $\|x, z\|=\|y, z\|=1, \quad x \neq y$ and $z \notin V(x, y)$. Then $x, y \in S_{z}(0,1)$ and since $\alpha=\beta=\frac{1}{2}$ we get that $\left\|\frac{x+y}{2}, z\right\|<1$, which according to theorem 1 means that $L$ is strictly convex.

Conversely, let's assume that there exist $x, y \in S_{z}(0,1), x \neq y$ and $\alpha, \beta>0$, $\alpha+\beta=1$ such that $\|\alpha x+\beta y, z\|=1$. The latter implies that there exist $x, y, z \in L$ such that $\|x, z\|=\|y, z\|=1, x \neq y$ and $\alpha, \beta>0, \alpha+\beta=1$ such that

$$
\|\alpha x+\beta y, z\|=\|\alpha x, z\|+\|\beta y, z\| \text {. }
$$

Since Lemma 1,

$$
\|t(\alpha x)+s(\beta y), z\|=t\|\alpha x, z\|+s\|\beta y, z\|,
$$

holds true for all $t, s \geq 0$. For $t=\frac{1}{2 \alpha}, s=\frac{1}{2 \beta}$, in the last equality, we get that there exist $x, y, z \in L$ such that

$$
\|x, z\|=\|y, z\|=1, x \neq y \text { and }\left\|\frac{x+y}{2}, z\right\|=1 .
$$

The latter, according to theorem 1 , means that the space $L$ is not strictly convex.

In the purpose of the next characterization of strictly convex 2-normed space we will use the extremal points of the convex sets. Let $C$ be a convex set into 2-normed space $L$. The point $z \in C$ is said to be an extremal (end) point for the set $C$ if $z=t x+(1-t) y$, for some $t \in(0,1)$ and some $x, y \in C$ implies $x=y$.

Theorem 4. 2- normed space $L$ is strictly convex if and only if for each $z \in L$ each point of the unit sphere with respect to $z$ is an extremal point of the closed unit ball with respect to $z$.

Proof. Let $L$ be strictly convex space. It will be proven that each point of the set $S_{z}(0,1)$ is an extremal point of the set $B_{z}[0,1]$. Let $u \in S_{z}(0,1)$ and let $u=t x+(1-t) y$ for some $t \in(0,1)$ and some $x, y \in B_{z}[0,1]$. Thereby $u \in S_{z}(0,1)$, it is true that $\|u, z\|=1$ and since $x, y \in B_{z}[0,1]$ we get that $\|x, z\| \leq 1,\|y, z\| \leq 1$. It will be proven that $\|x, z\|=\|y, z\|=1$ holds true. Indeed, in otherwise it holds that

$$
1=\|u, z\|=\|x+(1-t) y, z\| \leq t\|x, z\|+(1-t)\|y, z\|<1
$$


which is contradictory. Thus, $\|x+y, z\| \unlhd\|x, z\|+\|y, z\|=2$. At the end, it will be proven that $\|x+y, z\|=2$ holds. Indeed, in otherwise it holds that

$$
\begin{aligned}
1 & =\|u, z\|=\|t u+(1-t) u, z\| \\
& =\|t[t x+(1-t) y]+(1-t)[t x+(1-t) y], z\| \\
& =\left\|t^{2} x+t(1-t)(x+y)+(1-t)^{2} y, z\right\| \\
& <t^{2}+2 t(1-t)+(1-t)^{2}=1,
\end{aligned}
$$

which is contradictory. So, $\|x, z\|=\|y, z\|=\left\|\frac{x+y}{2}, z\right\|=1$ and since $L$ is strictly convex space, we get that $x=y$, i.e. $u$ is an extremal point of the closed unit ball with respect to $z$.

Conversely, let's assume that $\|x, z\|=\|y, z\|=\left\|\frac{x+y}{2}, z\right\|=1$ and $z \notin V(x, y)$, for $x, y, z \in L$. Thus, the point $u=\frac{1}{2} x+\frac{1}{2} y$ is on the unit sphere with respect to $z$. Therefore, it is an extremal point of the closed unit ball with respect to $z$, that is $x=y$, i.e. $L$ is strictly convex space.

Example 3. Over the vector space $\mathbf{C}_{[0,1]}$, of continuous functions on the interval $[0,1]$, the function $\|\cdot, \cdot\|: \mathbf{C}_{[0,1]} \times \mathbf{C}_{[0,1]} \rightarrow \mathbf{R}$ defined as

$$
\|x, y\|=\max _{s, t \in[0,1]}|| \begin{array}{ll}
x(t) & x(s) \\
y(t) & y(s)
\end{array}|| .
$$

is a 2-norm. So, $\left(\mathbf{C}_{[0,1]},\|\cdot, \cdot\|\right)$ is 2-normed space. The functions

$$
x(t)=1, y(t)=1-t, z(t)=t^{2} \in \mathbf{C}_{[0,1]}
$$

satisfy

$$
\begin{aligned}
& \|x, z\|=\max _{s, t \in[0,1]}|| \begin{array}{cc}
1 & 1 \\
t^{2} & s^{2}
\end{array}||=\max _{s, t \in[0,1]}\left|s^{2}-t^{2}\right|=1 \text { and } \\
& \|y, z\|=\max _{s, t \in[0,1]}|| \begin{array}{cc}
1-t & 1-s \\
t^{2} & s^{2}
\end{array}||=\max _{s, t \in[0,1]}\left|s^{2}-t^{2}+s t^{2}-t s^{2}\right|=1 .
\end{aligned}
$$

Therefore, $x, y \in S_{z}(0,1)$. Further the functions $u(t)=\frac{1}{2} x(t)+\frac{1}{2} y(t)=1-\frac{t}{2}$ satisfy

$$
\|u, z\|=\max _{s, t \in[0,1]}|| \begin{array}{cc}
1-\frac{t}{2} & 1-\frac{s}{2} \\
t^{2} & s^{2}
\end{array}||=\max _{s, t \in[0,1]}\left|s^{2}-t^{2}-\frac{1}{2} t s^{2}+\frac{1}{2} s t^{2}\right|=1 .
$$


Therefore $u \in S_{z}(0,1)$ and since it is not an extremal point of $B_{z}[0,1]$ we deduce that the space $\left(\mathbf{C}_{[0,1]},\|\cdot, \cdot\|\right)$ is not strictly convex space.

In the purpose of the next characterization of strictly convex 2-normed space we will introduce the term of minimal point with respect to the set $M \subseteq L$.

The point $v \in L$ is said to be minimal point with respect to the set $M$ if $\|u-m, z\| \leq\|v-m, z\|, \quad z \notin V(u-M)=V(\{u-m \mid m \in M\}), \quad u, z \in L$ and for each $m \in M$ implies that $u=v$.

Theorem 5. 2-normed space $L$ is strictly convex if and only if for all $x, y \in L$ the points of the segment $[x, y]=\{t x+(1-t) y \mid t \in[0,1]\}$ are minimal with respect to the set $\{x, y\}$.

Proof. Let $L$ be strictly convex space. Clearly, the points $x$ and $y$ are minimal with respect to the set $\{x, y\}$. Thus, let $v_{t}=t x+(1-t) y, t \in(0,1)$ be any point of the opened line segment $[x, y]$. Let's assume that for some $u \in L$ and $z \notin V(x, y)$

$$
\begin{aligned}
& \|u-x, z\| \leq\left\|v_{t}-x, z\right\|=(1-t)\|x-y, z\| \\
& \|u-y, z\| \leq\left\|v_{t}-y, z\right\|=t\|x-y, z\| .
\end{aligned}
$$

hold true.

Further, the inequalities (5) and (6) imply

$$
\begin{aligned}
\|x-y, z\| & \leq\|x-u, z\|+\|u-x, z\| \\
& \leq(1-t)\|x-y, z\|+t\|x-y, z\| \\
& =\|x-y, z\| .
\end{aligned}
$$

Thus, the following equalities hold true

$$
\|u-x, z\|=(1-t)\|x-y, z\|,\|u-y, z\|=t\|x-y, z\|
$$

and since $t \in(0,1)$ and $z \notin V(x-u, y-u)$, theorem 1 implies that $u=t x+(1-t) y=v_{t}$. That is, $v_{t}$ is minimal point with respect to the set $\{x, y\}$. Conversely, let for all $a, b \in L$ the points of the segment $[a, b]$ be minimal for the set $\{a, b\}$. If $\|x, z\|=\|y, z\|=\left\|\frac{x+y}{2}, z\right\|=1$ and $z \notin V(x, y)$, then

$$
\begin{aligned}
& \|0-x, z\|=\|x, z\|=\left\|\frac{x+y}{2}, z\right\| \leq\left\|\frac{1}{2} x+\frac{1}{2}(-y)-x, z\right\|, \\
& \|0-(-y), z\|=\|y, z\|=\left\|\frac{x+y}{2}, z\right\| \leq\left\|\frac{1}{2} x+\frac{1}{2}(-y)-(-y), z\right\|
\end{aligned}
$$

and $z \notin V(x, y)=V(0-x, 0-(-y))$. 
But, the point $\frac{1}{2} x+\frac{1}{2}(-y)$ belongs to a segment $[x,-y]$. That is, it is minimal with respect to the set $\{x,-y\}$. So,

$$
\frac{1}{2} x+\frac{1}{2}(-y)=0
$$

i.e. $x=y$. That is, $L$ is strictly convex.

\section{CONFLICT OF INTEREST}

No conflict of interest was declared by the authors.

\section{AUTHOR'S CONTRIBUTIONS}

All authors contributed equally and significantly to writing this paper. All authors read and approved the final manuscript.

\section{References}

[1] Y. J. Cho, K. S. Ha and S. W. Kim, Strictly Convex Linear 2-Normed Spaces, Math. Japonica, Vol. 26 (1981), 475-478.

[2] Y. J. Cho, B. H. Park and K. S. Park, Strictly 2-convex Linear 2-normed Spaces, Math. Japon. Vol. 27 (1982), 609-612.

[3] C. Diminnie, S. Gähler and A. White, 2-Inner Product Spaces, Demonstratio Mathematica, Vol. VI, No 1 (1973), 525-536.

[4] C. Diminnie, S. Gähler and A. White, 2-Inner Product Spaces II, Demonstratio Mathematica, Vol. X, No 1 (1977), 169-188.

[5] C. Diminnie and S. Gahler, Some geometric results concerning strictly 2convex 2-normed spaces, Math. Seminar Notes, Kobe Univ., Vol. 6 (1978), 245-253.

[6] C. Diminnie, S. Gahler and A. White, Strictly Convex Linear 2-Normed Spaces, Math. Nachr. Vol. 59 (1974), 319-324.

[7] C. Diminnie, S. Gahler and A. White, Remarks on Strictly Convex and Strictly 2-Convex 2-Normed Spaces, Math.Nachr. Vol. 88 (1979), 363372.

[8] C. Diminnie and A. White, Strict convexity in topological vector spaces, Math. Japon. Vol. 22 (1977), 49-56.

[9] R. Ehret, Linear 2-normed Spaces, Doctoral Diss., Saint Louis Univ., 1969.

[10] R. W. Freese, Y. J. Choand S. S. Kim, Stryctly 2-convex linear 2-normed spaces, J. Korean Math. Soc. Vol. 29 (1992), 391-400. 
[11] S. Gähler, Lineare 2-normierte Räume, Math. Nachr. Vol. 28 (1965), 1-42.

[12] K. S. Ha, Y. J. Cho and A. White, Strictly convex and strictly 2-convex linear 2- normed spaces, Math. Japon. Vol. 33 (1988), 375-384.

[13] K. Iseki, On non-expansive mappings in strictly convex 2-normed spaces, Math. Seminar Notes, Kobe Univ., Vol. 3 (1975), 125-19.

[14] K. Iseki, Mathematics in 2-normed spaces, Math. Seminar Notes, Kobe Univ., Vol. 6 (1976), 161-176.

[15] I. V. Istrăţescu, Strict convexity and complex strict convexity (theory and applications), Marcel Dekker, New York - Basel, 1984.

[16] A. Khan and A. Siddiqi, Characterization of Strictly Convex Linear 2-normed Spaces in Therms of Duality Bimappings, Math. Japonica Vol. 23, No. 1, (1978).

[17] S. Mabizela, A characterization of strictly convex linear 2-normed spaces, Questiones Math. Vol. 12 (1989), 201-204.

[18] A. Malčeski, $l^{\infty}$ as a n-normed space, Matematički bilten, Vol. 21 (XXI) (1997), 103-110.

[19] A. Malčeski and R. Malčeski, $L^{1}(\mu)$ as a $n$-normed space, Annuaire des l'institut des mathematiques, Vol. 38 (1997), 23-29.

[20] A. Malčeski and R. Malčeski, $L^{\mathrm{p}}(\mu)$ as a 2-normed space, Matematički bilten, Vol. 29 (XL) (2005), 71-76.

[21] R. Malčeski, Strong convex $n$-normed spaces, Macedonian Academy of Sciences and Arts, Contributions, Vol. XVIII 1-2 (1997), 39-57.

[22] R. Malčeski, Strong $n$-convex $n$-normed spaces, Matematički bilten, Vol. 21 (XXI) (1997), 81-102.

[23] R. Malčeski and K. Anevska, Strictly convex in quasi 2-pre-Hilbert space, IJSIMR, e-ISSN 2347-3142, p-ISSN 2346-304X, Vol. 2, Issue 07 (2014), 668-674.

[24] R. Malčeski, Lj. Nasteski, B. Načevska and A. Huseini, About the strictly convex and uniformly convex normed and 2-normed spaces, IJSIMR, eISSN 2347-3142, p-ISSN 2346-304X, Vol. 2, Issue 06 (2014), 603-610

[25] A. White, 2-Banach Spaces, Math. Nachr. Vol. 42 (1969), 43-60.

\footnotetext{
${ }^{1)}$ University Euro-Balkan, Skopje, Macedonia

E-mail address: samoil.malcheski@gmail.com

${ }^{2)}$ Faculty of informatics, FON University, Skopje, Macedonia E-mail address: risto.malceski@gmail.com

${ }^{3)}$ Faculty of informatics, FON University, Skopje, Macedonia E-mail address: anevskak@gmail.com
} 